\title{
Linx
}

Revue des linguistes de l'université Paris X Nanterre

$76 \mid 2018$

Dire l'humain : les noms généraux dénotant les

humains

\section{Homme, personne, être humain : trois noms d'humains généraux en français et en bulgare}

\section{Vassil Mostrov et Angelina Aleksandrova}

\section{(2) OpenEdition}

\section{Journals}

Édition électronique

URL : http://journals.openedition.org/linx/2531

DOI : $10.4000 /$ linx.2531

ISSN : 2118-9692

Éditeur

Presses universitaires de Paris Nanterre

Édition imprimée

Date de publication : 31 juillet 2018

Pagination : 133-162

ISSN : 0246-8743

Référence électronique

Vassil Mostrov et Angelina Aleksandrova, « Homme, personne, être humain : trois noms d'humains généraux en français et en bulgare », Linx [En ligne], 76 | 2018, mis en ligne le 31 janvier 2019, consulté le 19 avril 2019. URL : http://journals.openedition.org/linx/2531 ; DOI : 10.4000/linx.2531

Ce document a été généré automatiquement le 19 avril 2019

Département de Sciences du langage, Université Paris Ouest 


\title{
Homme, personne, être humain : trois noms d'humains généraux en français et en bulgare
}

\author{
Vassil Mostrov et Angelina Aleksandrova
}

\section{Introduction}

1 Notre travail propose d'aborder les noms d'humains généraux français (désormais NHGfr) dans une perspective comparative avec le bulgare. Dans la mesure où il n'existe pas de description approfondie des noms d'humains pour cette langue (cf. pourtant Venkova, 1997) nous poursuivons un double objectif : a) répertorier les NHGbg afin d'offrir un panorama lexicologique fondé sur leurs spécificités morphologiques, syntaxiques et sémantico-référentielles et b) expliciter et caractériser les contraintes observées dans la traduction de trois NHGfr, à savoir homme, personne et être humain. Le choix de restreindre l'étude à cet échantillon est triplement motivé. Premièrement, parmi les NHGfr, ces trois lexèmes ont ceci en commun d'être tous des substantifs (pour ce qui est d'humain, il est avant tout un adjectif et n'accède à la catégorie nominale que par conversion), de varier en nombre (ce n'est pas le cas de gens, par exemple) et de dénoter spécifiquement les humains (même si individu est aussi un NHG, sa référence à l'humain n'est que secondaire dans les traitements lexicographiques ${ }^{1}$ ). Puis il s'agit pour nous de poursuivre et compléter la description de ces lexèmes déjà entamée au sein de l'équipe NHUMA (cf. Cappeau \& Schnedecker, 2014 pour une étude diamésique et la comparaison avec gens ; Mihatsch, 2015 pour la comparaison avec l'allemand) et enfin, plus particulièrement, d'approfondir la perspective comparative avec le bulgare (Aleksandrova \& Mostrov, 2016).

2 Au niveau méthodologique, nous nous appuyons à la fois sur des ressources lexicographiques (cf. bibliographie) et des données de corpus. Nous avons sélectionné un échantillon d'occurrences à partir de la base de données OPUS (Tiedermann, 2009) ${ }^{2}$ 
provenant de trois domaines différentes: textes médicaux (corpus MED) ${ }^{3}$, politiques (corpus EUR) ${ }^{4}$ et de sous-titres (corpus SUB) ${ }^{5}$.

Du point de vue global, l'échantillon comporte au total 749 occurrences des différentes formes des lexèmes suivants : être humain, homme, humain, individu, personne, gens. L'unité d'analyse est la phrase d'accueil de l'item avec un cotexte plus ou moins large selon les corpus et sa traduction en bulgare. La répartition des sous-corpus est la suivante :

7 La Figure 1 illustre la répartition de chaque forme de l'échantillon NHGfr selon le type de corpus.

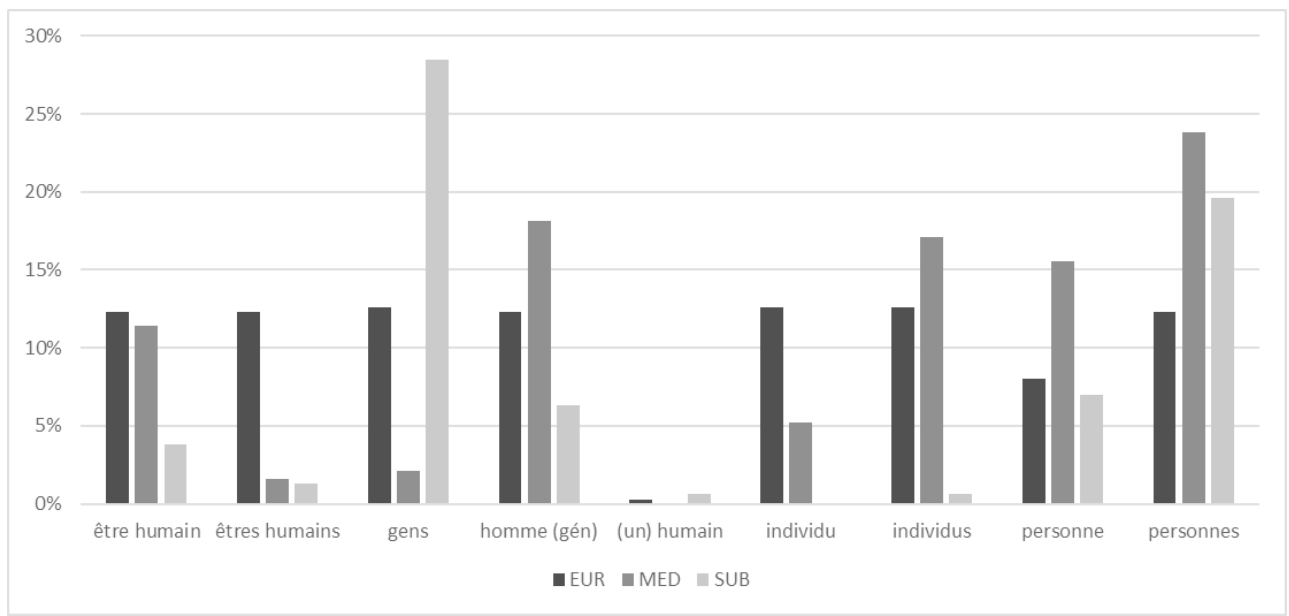

Figure 1. Répartition des NHGfr par Corpus

8 L'inégalité des sous-corpus est avant tout le résultat de la phase de nettoyage (élimination des doublons, des inadéquations dans les traductions, etc.). Même si les données relèvent majoritairement de domaines spécialisés (notamment médecine et droit ${ }^{6}$ ), l'emploi des NHG ne peut pas être considéré comme relevant intrinsèquement d'un langage technique particulier (par ailleurs, dans EUR, certaines occurrences se rapprochent du langage courant).

Le traitement du corpus a été fait à l'aide d'un tableau analytique dans lequel nous avons enregistré, manuellement, différentes informations (morphologiques, syntaxiques, lexicales, etc.) pour chaque occurrence et aussi bien en français qu'en bulgare.

Dans ce qui suit, nous nous focalisons sur l'analyse d'une partie des données ainsi obtenues, notamment pour personne, être humain, et homme (au sens général, synonyme d' être humain, désormais homme-g). Les Figures 2 et 3 indiquent la proportion respectivement des lemmes et des formes présentes dans notre corpus. Notons l'absence dans tout le corpus du pluriel hommes en lecture générique, comme dans Les hommes sont mortels. 


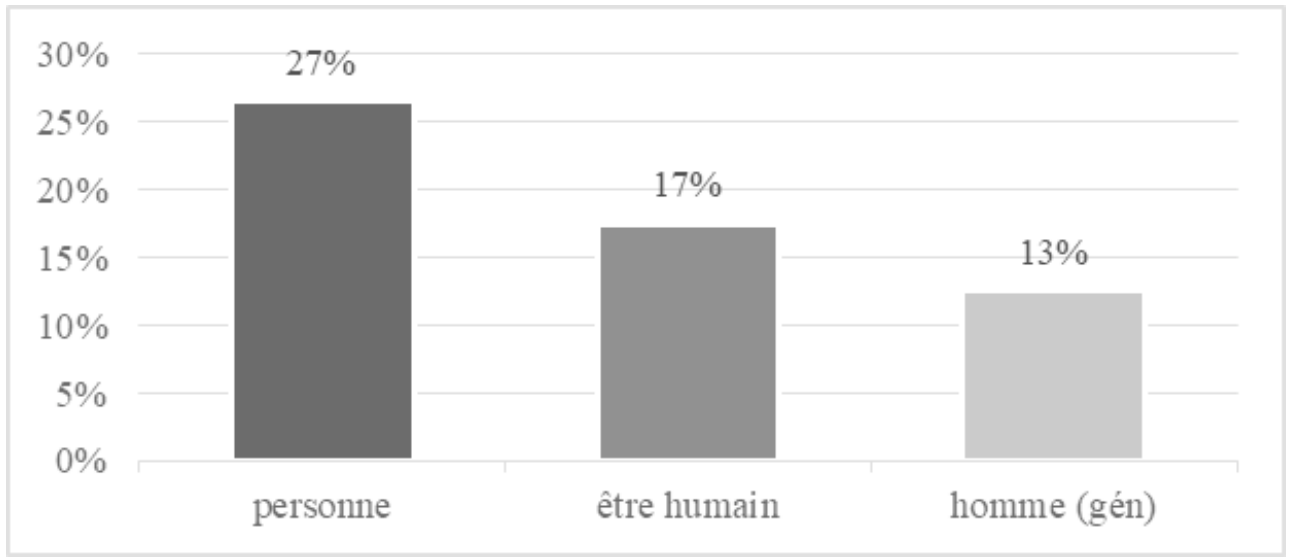

Figure 2. \% NHGfr-lemme (sur 749 occ.)

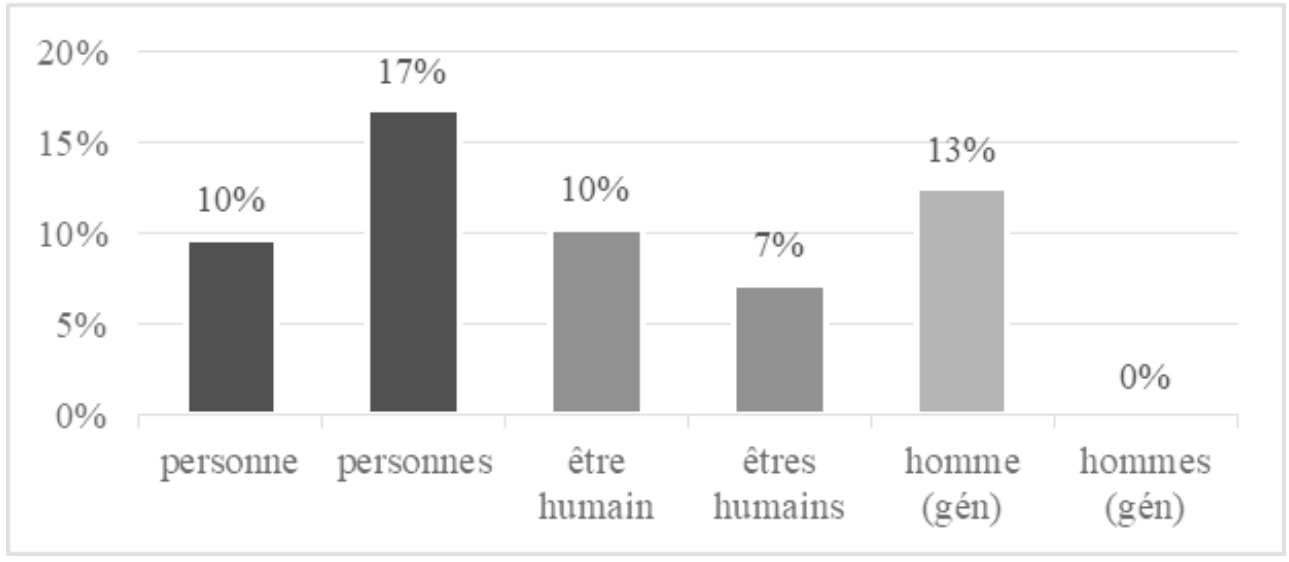

Figure 3. \% NHGfr-forme (749 occ.)

11 Enfin, la Figure 4 offre une troisième vue globale sur les données avec la répartition de chaque lemme par sous-corpus. Il est à noter que personnes constitue presque un quart des occurrences du corpus MED ( $40 \%$ avec le singulier personne ${ }^{7}$ ).

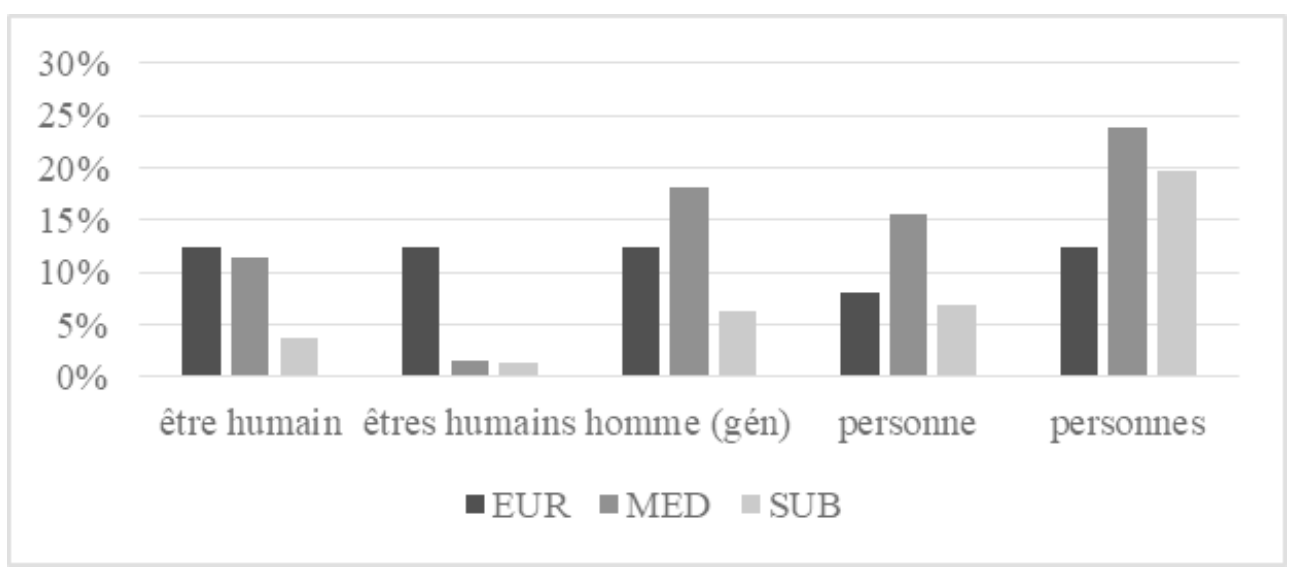

Figure 4. Répartition NHGfr par Corpus 


\section{Panorama des NHG-bg}

\subsection{Du point de vue morphosyntaxique}

Il convient de distinguer, parmi les NHG-bg, ceux qui enregistrent une variation en nombre (1) et les pluralia tantum (2) :

(1)

(a) човек /chovek/8 'homme', 'humain', parfois 'on'

(b) чОВЕшко СъщЕСТВо /choveshko sashtestvo/ 'être humain'

(c) лице /litse/ 'personne', parfois 'individu'

(d) индивид /individ/ 'individu'

(2)

(e) Xopa /hora/ 'gens'

(f) души /doushi/ 'personnes' (pour dénombrer les humains, > 2)

Dans ce qui suit, nous présenterons le choix des traductions en bulgare de homme et personne dans le Dictionnaire français-bulgare (2002), en fonction de la «polysémie » de ces deux NHG-fr. Notons que pour ce qui est de la polylexie être humain, elle ne fait pas l'objet d'un article à part, ce qui ne permet pas d'en proposer une description lexicographique complète.

\subsection{Traductions en bulgare des trois NHG-fr choisis}

\subsubsection{Homme $-\mathrm{g}$}

14 Il est bien connu qu'en français homme dénote aussi bien l'être humain en général ( homme-g) que l'être humain mâle (homme-m). En revanche, en bulgare on est en présence de deux lexèmes qui correspondraient respectivement à ces deux « sens » : ЧОВЕК (chovek) pour homme-g et мъЖ (maj) pour homme-m, ce qui justifie le traitement homonymique par le dictionnaire bilingue consulté :

HOMME 1 : ЧОВЕК.

$(\mathrm{A})^{10}(=$ « terme de taxinomie animale » $(\mathrm{PR}))$

Homme primitif: ПЪРВОБИТЕН ЧОВЕК

Homme des cavernes : ПЕЩЕРЕН ЧОВЕК

(B) (= « être humain mâle adulte, caractérisé par une qualité » (PR))

Homme d'action : ЧОВЕК НА ДЕЙСТВИЕТО

Homme de bien: ПОРЯДЪЧЕН/ДОБЪР ЧОВЕК

Homme d'esprit : ДУХОВИТ ЧОВЕК

(C) (= « être humain mâle adulte, caractérisé par sa fonction » $(\mathrm{PR}))$

Homme d'affaires: БИЗНЕСмЕН 'businessman'

Homme de loi : ЮРИст 'juriste'

Homme de guerre : BOEHEH 'militaire'

HOMME 2 : Мъж

Homme viril: МъЖЕСТВЕН МЪЖ

Vêtements d'homme: МъЖКИ ДРЕХИ

Parler d'homme à homme: ГОВОРя КАТО МъЖ С МъЖ

Ne pleure pas, sois un homme!: НЕ ПЛАЧИ, БЪДИ МЪЖ!

D'emblée, ces données nous permettent de conclure que si maj (=homme 2) correspond clairement au "sens » homme-m, la situation concernant la correspondance chovek: homme-g est loin d'être parfaite. Sous HOMME 1, on est en présence de collocations où le 
$\mathrm{NH}$ homme signifie homme-m (homme de bien/d'action) selon les dictionnaires français ( $P R$, Lexis), et où il est pourtant traduit par chovek. Le traitement homonymique de homme, proposé par le dictionnaire bilingue, ne se fonde donc pas (entièrement) sur la distinction « NH général vs mâle », mais sur le choix du lexème traduit (chovek vs maj).

Il s'avère donc que si homme-g est toujours traduit par chovek (cf. HOMME 1 (A)), ce NH-bg peut également empiéter sur le terrain de homme-m et entrer de ce fait en concurrence avec le $\mathrm{NH}[+$ masc.] maj. Cela signifie que même si en bulgare il existe deux lexèmes qui couvrent les deux acceptions de homme, l'un des deux, à savoir chovek, présente la polysémie inhérente à homme, quoique pas de la même façon ${ }^{11}$.

Pour ce qui est de HOMME 1 - où domine le sens [+gen] ${ }^{12}$ qui est celui qui nous intéresse dans le cadre de ce travail - il est traduit, dans la plupart des cas, et sans surprise, par son « équivalent» bulgare chovek, mais il existe également des traductions par des $\mathrm{NH}$ spécifiques, surtout lorsqu'il s'agit de «fonctions» (C). Dans ce dernier cas, le NH-cible équivaut à la collocation-source toute entière, en "intégrant» donc le NH. Dans la terminologie qu'on a adoptée relativement à notre corpus, cela correspondrait à des traductions de type SPEC.

\subsubsection{Personne}

Le NHG-fr personne est d'un usage très fréquent, ce qui pourrait être expliqué (i) par son signifié de base très général et (ii) par le fait qu'il n'encode presque jamais le sexe ${ }^{13}$ (à la différence d'homme). Comme on le verra à travers l'analyse des données du corpus (section 3), le bulgare ne dispose pas d'un lexème unique équivalant aux multiples emplois de ce NHG-fr, ce qui est à la source de problèmes particuliers. Voici les traductions proposées par le dictionnaire bilingue :

PERSONNE : litze 'personne', lichnost 'personnalité', chovek 'homme'

(A) (= « individu en général » $(\mathrm{PR}))$

Une personne: ЕДИН ЧОВЕК

Quelques personnes : Няколко дУши

Groupe de personnes : ГРУПА ХОРА

Grandes personnes : ГОЛЕМИТЕ, ВЪЗРАСТНИТЕ

Il est venu avec cette personne : ТОЙ ДОЙДЕ С ТОВА ЛИЦЕ

Personne âgée : ВЪЗРАСТЕН ЧОВЕК

Par personne : HA ЧОВЕК

(B) (= « individu en particulier » : personnalité / apparence extérieure (PR))

Etre content de sa personne : ДОВОЛЕН СЪМ ОТ СОБСТВЕНАТА СИ ОСОБА

Je m'adresse à la personne (et non à l'employé) : ОБРЪЩАМ СЕ КЪМ ЧОВЕКА (НЕ КЪМ

СЛУЖЕБНОТО ЛИЦЕ)

Soigner sa personne : ГРИЖА СЕ ЗА СЕБЕ СИ

Être bien de sa personne ИЗГЛЕЖДАМ ДОБРЕ ФИЗИЧЕСКИ

En personne : Лично 'personnellement' (Il est venu en personne : ТОЙ дОЙДЕ ЛИчно)

(C) $(=$ « sens juridique » $(\mathrm{PR}))$

Personne civile, morale : ЮРИДИЧЕСКО ЛИЦЕ

Personne à la charge : ЛИЦЕ ИМАЩО ПРАВО НА ИЗДРЪЖКА

Erreur sur la personne : ЗАБЛУДА ПО ОТНОШЕНИЕ НА СУБЕКТА

Identité d'une personne : САмо личност

(D) (= « sens grammatical » $(\mathrm{PR}))$

Parler à la troisième personne : ГОВОРЯ В ТРЕТО ЛИЦЕ

Le dictionnaire propose trois équivalents bulgares, qui sont censés couvrir les différents sens de personne : лице (litze) pour les sens spécialisés (juridique et grammatical), личност 
(lichnost) pour la facette immatérielle (le «moi») et ЧОВЕК (chovek) qui semble correspondre à une présentation "globale» de l'être humain. Mais ces trois lexèmes n'épuisent pas tous les emplois de personne, ce dont témoignent les traductions de différentes collocations sous (A)-(D).

On a l'impression d'une relative régularité dans les traductions concernant le sens (A) (le plus général) d'un côté et les sens (C) et (D) (les plus spécialisés) de l'autre. Pour ce qui est de (A), c'est chovek (« homme ») qui est choisi et ses « variantes » au pluriel que sont hora et doushi (cf. infra pour leur distribution). À partir de là, on peut formuler l'hypothèse que chovek est le NHGbg le plus " général », le plus « neutre », puisqu'il correspond aussi bien à homme-g qu'au sens le plus général de personne. Quant à litze (correspondant à $\mathrm{C}$ et $\mathrm{D}$ ), il partage avec personne l'absence d'encodage du sexe (il est «neutre » quant au genre) et son emploi dans la langue courante, qui est très rare, est stylistiquement marqué : Dans Toi doide s tova litze (=Il est venu avec cette personne), il s'agit d'une personne dont la présence n'est, pour le moins, pas souhaitée.

21 En revanche, pour le sens (B), qui fait référence d'un côté à la facette immatérielle de l'être humain et de l'autre à son apparence physique, différentes stratégies de traduction sont adoptées. Si pour le premier cas il existe des équivalents bulgares (les $\mathrm{NH}$ lichnost et osoba notamment, mais chovek n'est pas exclu), pour le second le dictionnaire recourt soit à des pronoms (soigner sa personne : " soigner soi même» littéralement), soit à des adverbes, comme dans être bien de sa personne (littéralement "physiquement») et en personne (" personnellement »).

\subsection{3. Être humain}

Aussi bien dans les dictionnaires français que dans les dictionnaires bulgares, être humain n'est pas traité comme une polylexie. On retrouve la traduction par chovek, lichnost en quatrième position dans l'entrée être (subst.) (après 1. syshtestvo 'être' subst., 2. jivot 'existence' subst., 3. tvar ${ }^{14}$ 'être', dépréc.). La traduction est littérale : choveshko systestvo (sg i pl.). Le Tableau 1 fait la synthèse des traductions possibles répertoriées par les ressources lexicographiques bilingues pour homme, personne et être humain.

Tableau 1. Synthèse des traductions lexicographiques de homme, personne et être humain

Regardons à présent la situation telle qu'elle se présente d'après l'analyse des données de corpus.

\section{Régularités dans la traduction de homme, être humain et personne en bulgare : données du corpus}

Pour commencer, donnons un aperçu global des possibilités de traduction pour chaque lexème telles qu'elles ressortent du traitement de notre corpus. Cette section se focalise sur chaque forme, pour laquelle nous présentons sous forme de figures les traductions respectives et réparties par type de sous-corpus. La section suivante offrira un autre point de vue sur la situation en faisant la synthèse des contraintes observées.

Afin de faciliter l'interprétation des figures qui vont suivre, le lecteur se rapportera à la légende ci-dessous. Par commodité, désormais, nous ferons l'économie de l'utilisation du cyrillique : le renvoi aux NHGbg se fera par la transcription phonétique et leur traduction littérale sera indiquée entre guillemets. 
Légende

\begin{tabular}{|c|c|c|}
\hline BG & Transcription phonétique & FR \\
\hline ЧОВЕК & chovek & « homme», « humain» \\
\hline $\begin{array}{l}\text { Ч.С-ВО (ЧОВЕШКО } \\
\text { СЪЩЕСТВО) }\end{array}$ & choveshko sashtestvo & "être humain" \\
\hline $\begin{array}{l}\text { Ч. С-ВА (ЧОВЕШКИ } \\
\text { СЪЩЕСТВА) }\end{array}$ & choveshki sashtestva & «êtres humains» \\
\hline ЛИЦЕ & litze & $\begin{array}{l}\text { "personne», } \\
\text { litt. «visage» }\end{array}$ \\
\hline лиЦА & litza & $\begin{array}{l}\text { "personnes » } \\
\text { litt. «visages» }\end{array}$ \\
\hline индивиди & individi & « individus » \\
\hline XOPA & hora & "gens » \\
\hline души & doushi & "personnes» (=dénombrable) \\
\hline PRO & pronom & \\
\hline SPEC & $\begin{array}{l}\text { lexème spécifique et/ou changement de } \\
\text { catégorie grammaticale }\end{array}$ & \\
\hline 0 & le NHGfr n'est pas traduit & \\
\hline
\end{tabular}




\subsection{Homme-g}

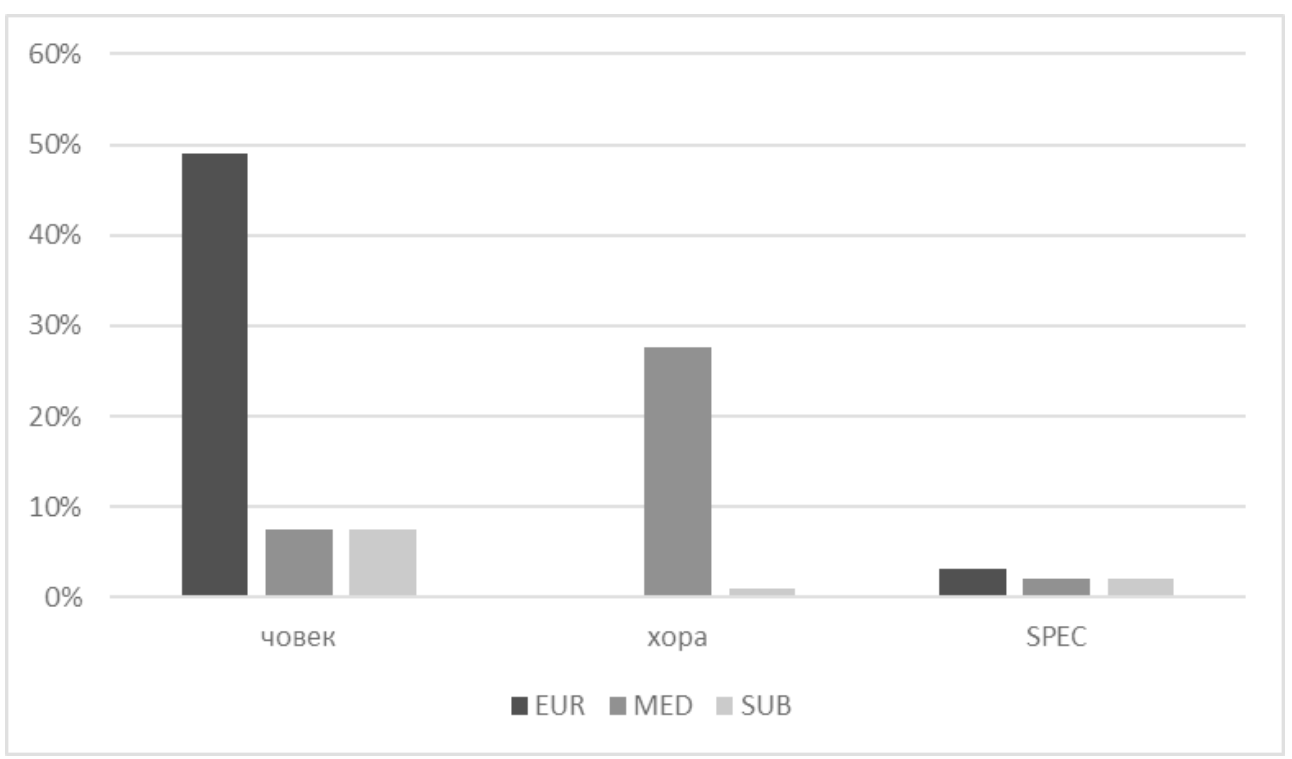

Figure 5. Homme : NHGbg

Homme-g est traduit principalement par chovek (sg. masc.), ou par hora (pl.), respectivement dans les exemples (3) et (4):

(3) Puis, l'homme fabriqua la machine à sa propre image.

ЧОВЕКЪТ СЪЗДАДЕ МАШИНАТА ПО СВОЕ СОБСТВЕНО ПОДОБИЕ.

(4) Il n'y a pas de données chez l'homme.

НЯМА ОПИТ ПРИ ХОРА.

Le grand nombre d'occurrences dans le corpus EUR (Figure 7 ci-dessous) s'explique par la nature des textes où il est souvent question des droits de l'homme, traduit littéralement par ПРАВАТА НА чОВЕКА (pravata na choveka). Étant donné le sens générique du lexème, la traduction par une forme de pluriel - hora - n'est pas surprenante. Si de prime abord, cet emploi est réservé au corpus médical, en réalité il n'en est rien puisque, pour ce même type de textes, des SP de type chez/pour l'homme sont traduits soit par pri/za choveka (PREP NHGbg, « chez/pour l'homme », cf. 5), soit par pri/za horata (PREP NHGbg « chez/pour les gens ", cf. 6).

(5)

L'échinococcose représente un danger pour l'homme.

ЕХИНОКОКОЗАТА (КУЧЕШКАТА ТЕНИЯ) ПРЕДСТАВЛЯВА ЗАПЛАХА ЗА ЧОВЕКА.

(6)

La biodisponibilité absolue du saquinavir coadministré avec du ritonavir n'a pas été établie chez l'homme.

ПРИ ХОРАТА АБСОЛЮТНАТА БИОНАЛИЧНОСТ НА САКВИНАВИР В КОМБИНАЦИЯ С РИТОНАВИР НЕ Е УСТАНОВЕНА. 


\section{2. Être(s) humain(s)}
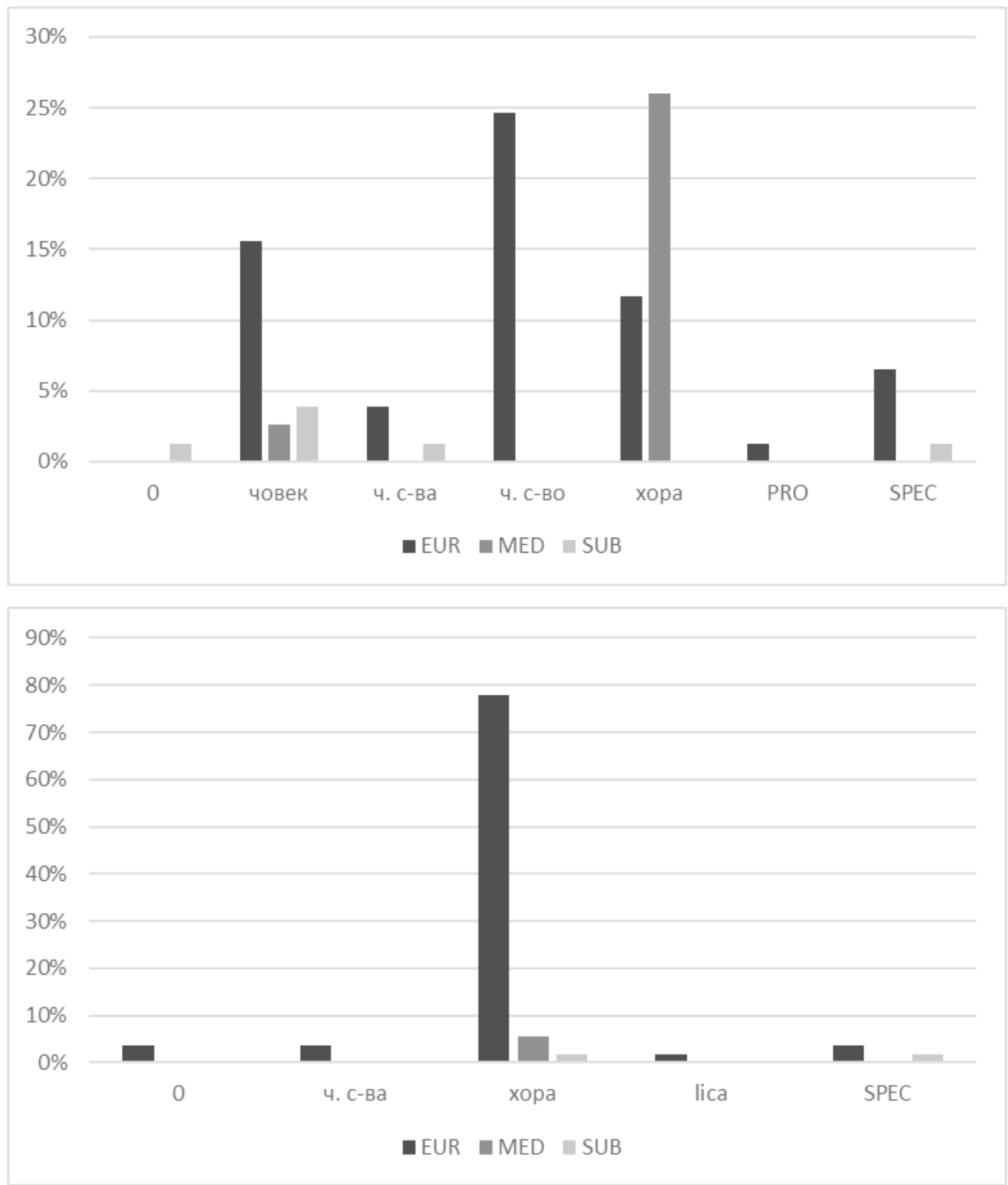

Figures $6 a$ et 6b. Traductions de «être humain » (en haut) et de « êtres humains » (en bas)

Les figures $6 \mathrm{a}$ et $6 \mathrm{~b}$ permettent de faire principalement trois observations concernant être (s) humain(s). Premièrement, la traduction de la forme au singulier est couverte principalement, dans l'ordre décroissant, par choveshko sashtestvo (subt. «être humain ») (7), chovek (subst. « un humain») - (8), et hora (subt. pl. « gens ») - (9-12).

(7)

La liberté n'est pas quelque chose qui se négocie; c'est un droit universel pour chaque être humain.

СВОБОДАТА НЕ ПОДЛЕЖИ НА ДОГОВАРЯНЕ, А Е УНИВЕРСАЛНО ПРАВО НА ВСЯКО ЧОВЕШКО СЪЩЕСТВО.

(8)

En raison des différences de physiologie osseuse entre l'être humain et le rat, la pertinence clinique de ces observations est probablement mineure.

ПОРАДИ РАЗЛИЧИЯТА ВЪВ ФИЗИОЛОГИЯТА НА КОСТИТЕ ПРИ ПЛЪХОВЕТЕ И ЧОВЕКА, КЛИНИЧНАТА ЗНАЧИМОСТ НА ТЕЗИ ДАННИ ВЕРОЯТНО Е МАЛКА. 
(9)

C'est un fait que, dans le sillage du 11 septembre 2001, aucun être humain ne considère que sa sécurité ou son intégrité physique est garantie.

ФАКТ Е, ЧЕ СЛЕД 11 СЕПТЕМВРИ 2001 Г. ХОРАТА НЕ ПРИЕМАТ СВОЯТА СИГУРНОСТ И ФИЗИЧЕСКА БЕЗОПАСНОСТ ЗА ДАДЕНОСТ.

(10)

Elles sont sûres, écologiques et moins nocives pour l'être humain.

ТЕ СА БЕЗОПАСНИ, ЩАДЯЩИ ОКОЛНАТА СРЕДА И СА ПО-МАЛКО ВРЕДНИ ЗА ХОРАТА.

(11) Les crises économiques révèlent des manques, causés par l'être humain.

ИКОНОМИЧЕСКИТЕ КРИЗИ РАЗКРИВАТ ДЕФИЦИТИ - ДЕФИЦИТИ, ПРИЧИНЕНИ ОТ ХОРА.

(12)

Puisque les risques pour l'être humain ne sont pas connus, Ciprofloxacine Hikma ne doit pas être administré pendant la grossesse.

ТЪЙ КАТО РИСКОВЕТЕ ЗА ХОРА СА НЕИЗВЕСТНИ, Ciprofloxacin Hikma НЕ ТРЯБВА ДА СЕ

ПРИЛАГА ПО ВРЕМЕ НА БРЕМЕННОСТ.

Deuxièmement, concernant la forme plurielle d'être humain, il convient de remarquer sa traduction majoritaire par le pluriel hora (dans la plupart des occurrences il est question de trafic d'êtres humains, dans le corpus EUR). Enfin, sous SPEC, nous avons observé soit le recours à une polylexie englobante (jivo sashtestvo, "vivant être", être vivant), soit l'emploi de l'ADJ correspondant avec changement du N-tête (race humaine). Nous aurons l'occasion d'y revenir.

\subsection{Personne(s)}

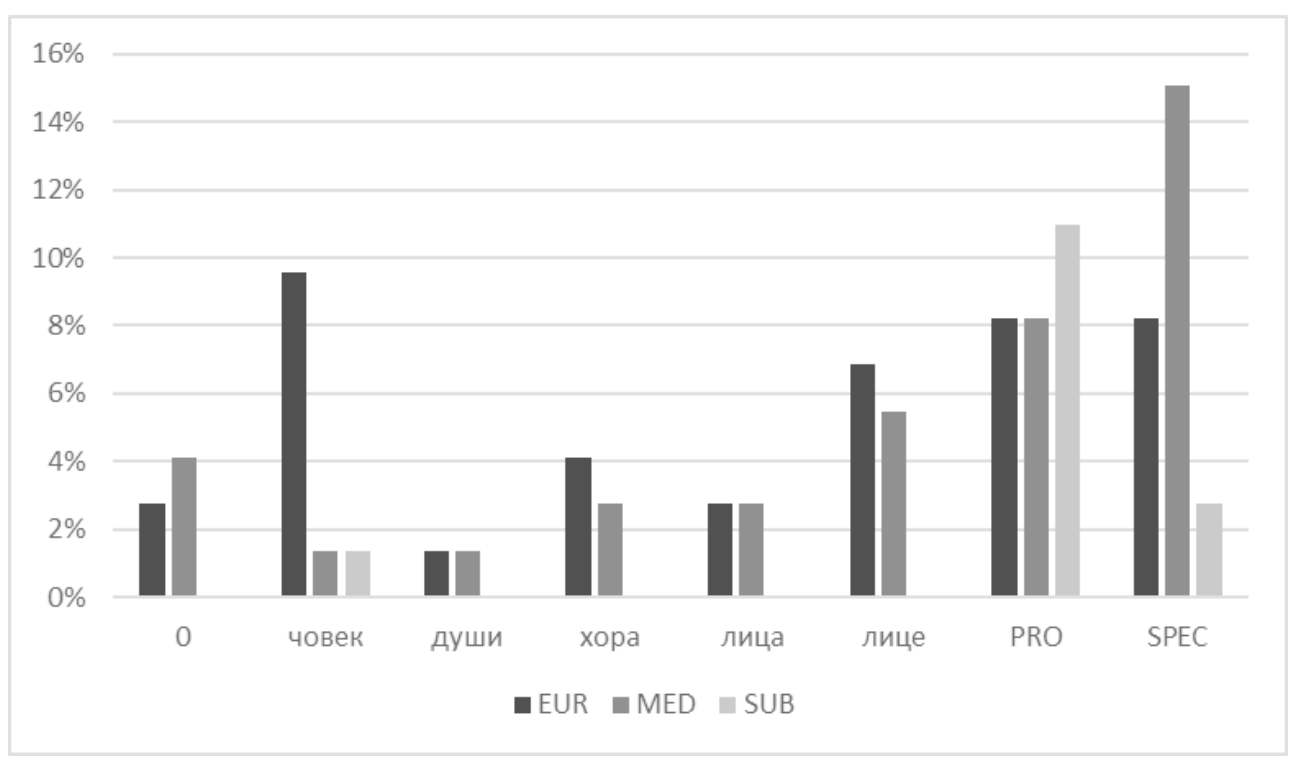




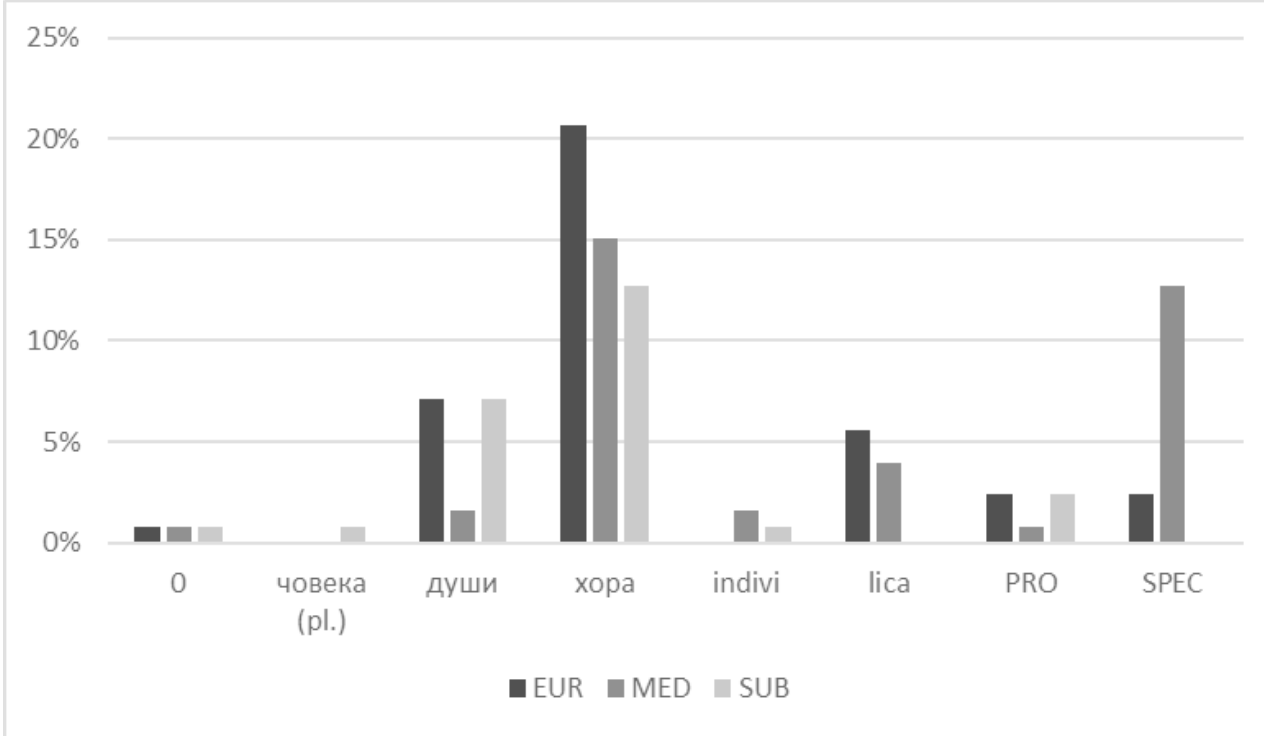

Figure 7 : Personne(s) : NHGbg personne dans le domaine juridique, ou plus généralement administratif. Dans ce cas, le lexème bulgare attendu est bien litze ( $\mathrm{pl}$. litza), dont l'emploi est en effet réservé au même type de langage.

(13)

Selon moi, toute personne doit avoir la garantie d'un revenu suffisant pour pouvoir mener une vie décente.

ПО МОЕ МНЕНИЕ, НА ВСЯКО ЛИЦЕ ТРЯБВА ДА СЕ ГАРАНТИРА ДОСТАТЪЧЕН ДОХОД, ЗА ДА МОЖЕ ДА ЖИВЕЕ ЕДИН ПРИЛИЧЕН ЖИВОТ.

(14)

Les autorités pakistanaises doivent faire quelque chose à propos du code pénal, qui prévoit la peine de mort pour toute personne reconnue coupable de blasphème.

ОРГАНИТЕ В ПАКИСТАН ТРЯБВА ДА НАПРАВЯТ НЕЩО ВЪВ ВРЪЗКА С НАКАЗАТЕЛНИЯ КОДЕКС, ПРЕДВИЖДАЩ СМЪРТНА ПРИСЪДА ЗА ВСИЧКИ ЛИЦА, КОИТО СА ОБВИНЕНИ И ОСЪДЕНИ ЗА БОГОХУЛСТВО.

31 Ce qui est moins attendu, en revanche, c'est que pour des données qui relèvent précisément de ce type de registre, cette traduction arrive seulement à la quatrième place (aussi bien pour personne que pour personnes) après les traductions par un NHGbg (notamment chovek « un humain») ou avec un changement lexical (sous SPEC), ou encore pronominales (PRO). Ces dernières seront examinées de plus près dans la section suivante.

De la Figure 7 il ressort, avant toute autre chose, que le spectre large d'emplois de personne ${ }^{15}$ en français n'a pas d'équivalent lexical en bulgare mais couvre des réalisations allant de la traduction «zéro " (NH non traduit, indiqué par 0 dans la figure) jusqu'aux emplois pronominaux (PRO) en passant par un dispositif lexical qui ne respecte pas forcément la variation en nombre (personne qui peut être traduit par des formes singulières ou plurielles).

En guise de bilan provisoire de cette section, nous invitons le lecteur à comparer le Tableau 1 qui faisait la synthèse des traductions possibles des trois NHGfr telles que 
proposées par les ressources lexicographiques avec le Tableau 2 ci-dessous, qui en fait de même mais d'après l'analyse de nos données de corpus.

Tableau 2. Synthèse des traductions (corpus) de homme, personne, et être humain

Insistons sur le fait qu'il n'est pas question pour nous de porter une critique au traitement lexicographique bilingue, mais plutôt d'apporter de nouvelles données ${ }^{16}$ pour son amélioration. Et c'est justement dans cette optique que la section suivante aura pour objectif d'expliciter et préciser les contraintes qui pèsent dans le choix d'une traduction adéquate des NHGfr choisis en bulgare.

\section{Synthèse des contraintes dans la traduction des NHG (français-bulgare)}

À la lumière des données que nous avons observées, nous dégageons quatre types de contraintes pour la traduction bulgare des NHG homme-g, être humain et personne.

\subsection{Contraintes de détermination}

Les contraintes de détermination sont essentiellement de deux types: l'un qui est étroitement lié à l'expression de la généricité, l'autre qui relève des spécificités morphosyntaxiques de certains items.

\subsubsection{Types de déterminants}

Commençons par ce dernier cas de figure, certainement plus facile à appréhender, puisqu'il s'agit d'un phénomène grammatical. Le type de déterminant en français est ici décisif, et on peut résumer la règle de la façon suivante :

- s'il s'agit d'un déterminant numéral (deux/dix/cent personnes), la traduction bulgare se fera exclusivement par doushi ("personnes»). Ce NHGbg a pour particularité d'être quasi-exclusivement employé au pluriel ${ }^{17}$, même s'il ne partage pas les propriétés d'un plurale tantum ;

- s'il s'agit d'un déterminant défini, indéfini, zéro ou d'un quantifieur indéfini, la traduction en bulgare se fait par le plurale tantum hora (équivalent fr. " gens »).

En effet, de ce point de vue, les deux lexèmes sont en distribution complémentaire en bulgare :

(15) deux / quelques personnes me l'ont dit ДВАМА/НЯКОЛКО *ХОРА/ДУШИ МИ ГО КАЗАХА

(16) beaucoup de personnes много *дУши/ХоРА

Par ailleurs, nous sommes enclins à dire que la traduction littérale d'être humain par choveshko sashtestvo (subst. «humain être »), qui n'apparait que dans le corpus EUR, est aussi liée à des phénomènes de détermination : dans $90 \%$ des cas, être humain apparaît avec un déterminant indéfini distributif (chaque être humain) ou totalisant (tout être humain), respecté par les traducteurs :

(17)

Ces droits sont inviolables et cela s'applique à chaque être humain.

ТЕЗИ ПРАВА СА НЕПРИКОСНОВЕНИ И ТОВА СЕ ОТНАСЯ ЗА ВСЯКО ЧОВЕШКО СЪЩЕСТВО.

(18) 
Le gouvernement guinéen persécute et tue quiconque ne partage pas les convictions politiques des dirigeants, privant ainsi la population de toute liberté, laquelle, nous le savons, est évidemment un droit inaliénable de tout être humain.

ПРАВИТЕЛСТВОТО НА ГВИНЕЯ ПРЕСЛЕДВА И УБИВА ВСЕКИ, ЧИИТО ПОЛИТИЧЕСКИ УБЕЖДЕНИЯ СЕ РАЗЛИЧАВАТ ОТ ТЕЗИ НА НЕГОВИТЕ ЛИДЕРИ, КАТО ПО ТОЗИ НАЧИН ЛИШАВА ХОРАТА ОТ ВСЯКАКЪВ ВИД СВОБОДА, КОЯТО, КАКТО ДОБРЕ ЗНАЕМ, ОЧЕВИДНО Е НЕПРИКОСНОВЕНО ПРАВО НА ВСЯКО ЧОВЕШКО СЪЩЕСТВО.

\subsubsection{Expression de la généricité} détermination. En français, mais c'est aussi vrai pour le bulgare, celle-ci peut avoir différentes réalisations. Concernant les NHG, nous avons vu que la généricité lexicale est enregistrée par les dictionnaires pour homme-g qui, dans cette acception, dénote la classe référentielle des hommes dans son ensemble. Au niveau syntagmatique, la généricité peut être induite aussi bien par l'indéfini que par l'article défini (singulier et pluriel) ${ }^{18}$ :

(19) un être humain, un humain, un homme est un être sensible.

(20) l'être humain, l'humain, l'homme est un être sensible.

(21) les êtres humains, les humains, les hommes sont des êtres sensibles.

41 En bulgare, la traduction privilégiée de homme-g se fait soit par le singulier chovek (subst. «un humain») soit par le pluriel hora (subst. «gens» au sens de les êtres humains, les hommes). Cette deuxième traduction par la forme du pluriel en bulgare est induite principalement par l'article zéro ou le défini singulier du SN français. Mais comme on le verra infra, le choix lexical peut varier en fonction des interférences au niveau contextuel.

\subsection{Contraintes morpho-syntaxiques}

Ce type de contraintes concerne surtout le NHGfr personne. Parmi ses traductions, nous observons une forte proportion de formes pronominales pour les occurrences où personne lui-même n'est pas en emploi pronominal ${ }^{19}$, cf. (22) et (23). En réalité, compte tenu du contexte, personne peut être traduit aussi bien par un PRO négatif (nikoj, comme dans personne n'est venu), qu'un PRO totalisant (dans (22), le PRO ВСЕКИ / vseki/, signifie littéralement "tout le monde»), un PRO indéfini (niakoj, comme dans demande à une personne de l'aide). Dans (23), il convient de souligner que la traduction bulgare ne tient pas compte de la subordonnée en si (dans ce cas la traduction adéquate aurait été par le pronom indéfini niakoj, «si quelqu'un qui ... »).

(22)

Toute personne qui regarde la télévision aux États-Unis à une heure de grande écoute peut constater les dégâts auxquels cette information peut donner lieu.

ВСЕКИ [PRO], КОЙТО ГЛЕДА ТЕЛЕВИЗИЯ В НАЙ-ГЛЕДАНОТО ВРЕМЕ В СЪЕДИНЕНИТЕ ЩАТИ МОЖЕ ДА РАЗБЕРЕ ДО КАКВИ ПРОБЛЕМИ МОЖЕ ДА ДОВЕДЕ ТОВА.

(23)

De l'autre côté, nous avons le parlement d'un État membre de l'UE qui a adopté une loi linguistique prévoyant que si une personne, en ce compris vous tous, n'appelle pas la capitale du pays par son nom dans la langue officielle du pays, Bratislava, mais utilise son nom allemand, Presburg, ou son nom hongrois, Pozsony, cette personne risque de payer une amende de 5000 euros.

СЛЕД ТОВА Е НАЛИЦЕ ПАРЛАМЕНТ НА ДЪРЖАВА-ЧЛЕНКА НА ЕС, КОЙТО НА 30 юНИ 2009 Г. ПРИЕ ЕЗИКОВ ЗАКОН, ПОСТАНОВЯВАЩ, ЧЕ ВСЕКИ [РRO], ВКЛЮЧИТЕЛНО ВСИЧКИ ВИЕ, КОЙТО НЕ НАРИЧА СТОЛИЦАТА НА ДЪРЖАВАТА С ИМЕТО Й НА ОФИЦИАЛНИЯ ЗА 
ДЪРЖАВАТА ЕЗИК, БРАТИСЛАВА, А ИЗПОЛЗВА НЕМСКОТО ПРЕСБУРГ ИЛИ УНГАРСКОТО

ПОЖОН, МОЖЕ ДА БЪДЕ ГЛОБЕН С 5000 ЕВРО.

43 Par ailleurs, exemple (24) à l'appui, dans les contextes où l'information sur le genre référentiel est pertinente (voire indispensable), la traduction pronominale de personne se révèle plus appropriée que ses équivalents lexicaux ${ }^{20}$. Rappelons que ce NHGfr n'est pas porteur d'informations de ce type: c'est le cotexte qui permet de préciser l'identité sexuelle du référent (enceinte). La traduction par le pronom ТАзи (tazi, PRO dém. « celle ») appelle une relative qui équivaut au complément adnominal (la traduction littérale de (24) est donc Une consultation médicale est nécessaire si celle qui va se faire vacciner est enceinte, essaie de tomber enceinte ou découvre qu'elle est enceinte au cours de la vaccination).

(24)

Si la personne à vacciner est enceinte, souhaite être enceinte ou découvre qu'elle est enceinte au cours du schéma de vaccination, parlez-en à votre médecin.

НЕОБХОДИМА Е КОНСУЛТАЦИЯ С ЛЕКАР, АКО ТАЗИ, КОЯТО ЩЕ СЕ ВАКСИНИРА Е БРЕМЕННА, ОПИТВА СЕ ДА ЗАБРЕМЕНЕЕ ИЛИ ЗАБРЕМЕНЕЕ В ХОДА НА ВАКСИНИРАНЕТО.

\subsection{Contraintes sémantico-référentielles}

Les contraintes que nous qualifions de sémantico-référentielles, et qui font partie des occurrences étiquetées sous SPEC dans les figures supra, conditionnent la traduction des NHGfr soit par un NH sémantiquement plus spécifique, soit par un changement de catégorie grammaticale. À ce niveau nous observons une différence très nette entre les traductions par un adjectif relationnel de être humain et homme-g d'une part, et personne, d'autre part.

Indépendamment de la fonction syntaxique en français, homme-g et être humain (sg. et pl.), donnent lieu à une traduction systématique par човЕшкИ /choveshki/ (humain, ADJ, dérivé de chovek, au sens de " propre à l'espèce humaine »), cf. (25)-(27). Par ailleurs pour homme-g sous SPEC, et cela pour les trois corpus, nous notons exclusivement des traductions par ce même adjectif (précisons que le bulgare permet la traduction du complément adnominal par l'adjectif: ЧОВЕШКИ ПРАВА, /choveshki prava/ litt. «droits humains $»^{21}$. De son côté, personne est traduit par личен (/lichen/, ADJ, "propre à l'individu », personnel), cf. (27), jamais par « humain ».

(25)

Le vardénafil est un inhibiteur puissant et sélectif de la phosphodiestérase de type 5 (PDE5), la principale forme de PDE présente dans les corps caverneux de l'homme.

ВАРДЕНАФИЛ Е МОЩЕН И СЕЛЕКТИВЕН ИНХИБИТОР НА СGМР СПЕЦИФИЧНАТА ФОСФОДИЕСТЕРАЗА ТИП 5 (РDЕ5), НАЙ-ВАЖНАТА ФОСФОДИЕСТЕРАЗА В ЧОВЕШКИЯ КОРПУС КАВЕРНОЗУМ.

(26)

Malgré nos différences politiques, et nonobstant ses carences manifestes en tant qu' être humain, je pense qu'on peut vraiment lui faire confiance là-dessus et je le soutiens.

ВЪПРЕКИ ПОЛИТИЧЕСКИТЕ НИ РАЗЛИЧИЯ И НЕЗАВИСИМО ОТ СЪВСЕМ ОЧЕВИДНИТЕ МУ ЧОВЕШКИ НЕСЪВЪРШЕНСТВА, АЗ СМЯТАМ, ЧЕ ТОЙ Е МНОГО СЕРИОЗЕН ПО ТОЗИ ВЪПРОС И ГО ПОДКРЕПЯМ.

(27)

La sécurité d'une personne ne peut pas, et ne devrait pas, s'arrêter aux frontières. ЛИЧНАТА [ADJ, « personnelle »] БЕЗОПАСНОСТ [N, « sécurité »] НЕ МОЖЕ И НЕ ТРЯБВА ДА ЗАВИСИ ОТ НАЦИОНАЛНИ ГРАНИЦИ. 
Par ailleurs, contrairement à être humain et homme-g, les traductions des SN expansés avec personne en $\mathrm{N}$-tête se font par des lexèmes sémantiquement plus spécifiques qui correspondent au modifieur français. Autrement dit, comme dans l'exemple (28), les contextes où personne a un emploi qui peut être qualifié d' "hyperonymique», la traduction bulgare préférera le lexème spécifique correspondant au modifieur (sans emploi), à savoir l'adjectif nominalisé bezraboten (fr. chômeur). Même si le bulgare permet la récupération d'un NHG support, qui dans ce cas précis serait litza (bezrabotni litza), elle parait superflue.

(28)

Monsieur le Président, il y a, à l'heure actuelle, 2,46 millions de personnes sans emploi au Royaume-Uni.

Г-Н ПРЕДСЕДАТЕЛ, В МОМЕНТА В ОБЕДИНЕНОТО КРАЛСТВО ИМА 2460000 БЕЗРАБОТНИ.

Les occurrences où personne est traduit par le pluriel hora peuvent nécessiter une restructuration phrastique. Dans (29) par exemple, la structure corrélative (d'une ... l'autre ) prend la forme d'un SN-pl. avec le modifieur adjectival différents (litt. «les différents gens »).

(29)

Monsieur le Président, la conviction religieuse est une affaire personnelle dont le sens diffère d'une personne à l'autre.

Г-Н ПРЕДСЕДАТЕЛ, РЕЛИГИОЗНИТЕ УБЕЖДЕНИЯ СА ЛИЧЕН ВЪПРОС, КОЙТО ИМА РАЗЛИЧНО СЪДЪРЖАНИЕ ЗА [PREP] РАЗЛИЧНИТЕ [ADJ, « différents »] ХOPA [NHG-pl, « gens »].

Considérons maintenant les deux exemples suivants, illustrant la majorité des occurrences rencontrées. Dans (30) personne grippée est traduit par un ADJ nominalisé (le malade), et dans (31) une (seule) personne est traduit par un lexème spécifique, à savoir slujitel (« un (seul) employé »).

(30)

Une étude a également examiné l'utilisation de Tamiflu dans un contexte familial (277 familles), à la fois en termes de traitement de la personne grippée et en termes de traitement ou de prévention de la grippe chez les personnes en contact avec ce patient.

В ЕДНО ОТ ПРОУЧВАНИЯТА Tamiflu Е ПРИЛАГАН И ПРИ СЕМЕЙСТВА (277 СЕМЕЙСТВА) КАКТО ЗА ЛЕчениЕ НА Болния [Nadj, « le malade »] от [Prep, « de »] гРиП [N, « grippe »], ТАКА И ЗА ЛЕЧЕНИЕ ИЛИ ПРОФИЛАКТИКА ЗА ТЕЗИ, КОИТО СА БИЛИ В КОНТАКТ С НЕГО.

(31)

L'aéroport de Manchester, qui teste un des scanners, souligne que les images en noir et blanc ne sont pas pornographiques ou érotiques et ne sont visionnées que par une seule personne située dans une pièce éloignée.

ЛЕТИЩЕ МАНЧЕСТЪР, КЪДЕТО СЕ ИЗПРОБВА ЕДИН ОТ СКЕНЕРИТЕ, ИЗТЪКВА АРГУМЕНТА, ЧЕ ЧЕРНО-БЕЛИТЕ ИЗОБРАЖЕНИЯ НЕ СА ПОРНОГРАФСКИ ИЛИ ЕРОТИЧНИ И ЧЕ ГИ ВИЖДА ЕДИНЕДИНСТВЕН СЛУЖИТЕЛ [N, sg. « employé »] В ИЗОЛИРАНО ПОМЕЩЕНИЕ, СЛЕД КОЕТО ТЕ НЕЗАБАВНО СЕ УНИЩОЖАВАТ.

Ce fait s'explique par le sémantisme propre de chovek (cf. section 2) et l'incapacité de jouer le rôle d'un substantif support qui en résulte. Si dans le premier exemple, l'insertion de chovek est malvenue pour des raisons d'alourdissement de la structure syntagmatique (littéralement «traitement de la personne malade de grippe»), la structure phrastique peut évidemment aussi jouer un rôle. Dans l'exemple suivant, le SP (la violence perpétrée) à l'égard d'une personne est traduit littéralement par " (la violence faite) en raison des convictions de la victime».

(32) 
La violence perpétrée à l'égard d'une personne en raison de ses convictions est abominable et nous devons la dénoncer sans coup férir.

НАСИЛИЕТО, ИЗВЪРШЕНО ЗАРАДИ УБЕЖДЕНИЯТА НА ЖЕРТВАТА, Е ОТВРАТИТЕЛНО И БЕЗ

СЪМНЕНИЕ ЗАСЛУЖАВА НАШАТА КРИТИКА. tion quant au genre, peut référer, si le contexte l'exige, à un sexe féminin. Il en est de même de la forme plurielle dans (34) (cf. la présence du $\mathrm{N}$ grossesse), mais le traducteur bulgare a choisi le NH spécifique zheni (femme, pl.). Le NHGbg hora (gens) ne conviendrait pas ici, même s'il est souvent utilisé dans des collocations relevant du domaine médical. La contrainte ne semble donc pas liée au « registre », mais au fait que hora dénote des êtres humains simultanément des deux sexes. Par contre, cette contrainte de la «mixité» des sexes ne semble pas peser sur le NHG-bg litza (du moins quand ce $\mathrm{N}$ est au pluriel), qui aurait donc pu être choisi dans la traduction.

(34)

Les personnes utilisant Enbrel devraient éviter une grossesse pendant le traitement avec Enbrel .

ЖЕНИТЕ, ИЗПОЛЗВАЩИ Enbrel, НЕ ТРЯБВА ДА ЗАБРЕМЕНЯВАТ.

\subsection{Contraintes discursives}

type de contraintes couvre deux réalités qu'il convient de distinguer. Pour la première, le discours doit être compris au sens strict: environnement cotextuel plus ou moins proche du NH pivot. Pour la seconde, les contraintes discursives renvoient plutôt aux genres discursifs.

\subsubsection{Contraintes de l'environnement discursif}

Le premier cas de figure de ce type est exemplifié par la mise en relation de deux NHGfr en discours. Dans l'exemple suivant, l'emploi sinon «hyperonymique » du moins englobant de êtres humains impose une double contrainte. La première est sur la 
traduction de personnes qui, pour des raisons stylistiques, ne peut pas être assumée par le pluriel choveci (ce qui donnera *choveci kato choveshki sashtestva, structure tautologique " les humains en tant qu'êtres humains »). Dans ce cas, le choix se porte naturellement sur hora ( "gens ») dont le sémantisme ne met pas en exergue une interprétation « taxinomique ». La seconde impose la traduction littérale de êtres humains lui-même, qui permet d'insister justement sur la dimension humaine des êtres dans un contexte spécifique (le droit du travail). N’étant pas sémantiquement neutre, cette polylexie sert à "isoler" une facette.

(35)

De manière générale, nous devons continuer à nous concentrer sur les personnes en tant qu'êtres humains, ce qui est réalisable en protégeant les droits des travailleurs et le droit au travail.

КАТО ЦЯЛО Е НЕОБХОДИМО ДА СЪСРЕДОТОЧИМ ВНИМАНИЕТО СИ ВЪРХУ ХОРАТА КАТО

ЧОВЕШКИ СЪЩЕСТВА, А ТОВА МОЖЕ ДА СЕ ПОСТИГНЕ ЧРЕЗ ЗАЩИТА НА ПРАВАТА НА РАБОТНИЦИТЕ И ПРАВОТО НА ТРУД.

L'exemple suivant (36) illustre ce type de contraintes, où êtres humains est coordonné avec un NH spécifique (migrants) et est traduit par litza (subs. pl. « visages», p.ext. personnes, admin.). La traduction littérale de la coordination aurait instauré une relation de type hypo-hyperonymique entre migrant/être humain ${ }^{22}$ et aurait laissé sous-entendre que "les migrants sont une sorte d'êtres humains". Pour éviter cette interprétation, le traducteur a opéré un double choix : a) passer par une coordination de type [N-hyponyme et d'autres $\mathrm{N}$-hyperonyme ${ }^{23}$ afin de respecter le sens induit par la coordination en français (existence de deux classes référentielles : trafic de migrants, trafic d'êtres humains) et $b$ ) traduire êtres humains par le pluriel litza, qui n'instaure pas de relation inclusive entre les deux classes.

(36)

Je dois dire que nous avons proposé une assistance financière croissante à la Libye, mais il est évident qu'il faut que les autorités libyennes utilisent cette assistance pour améliorer la lutte contre le trafic de migrants et d'êtres humains sur leur territoire et pour renforcer les contrôles à leur frontière méridionale.

ТРЯБВА ДА КАЖА, ЧЕ ПРЕДЛОЖИХМЕ ДА УВЕЛИЧИМ ФИНАНСОВАТА ПОМОЩ ЗА ЛИБИЯ И Е ОЧЕВИДНО, ЧЕ ЛИБИЙСКИТЕ ОРГАНИ ТРЯБВА ДА ИЗПОЛЗВАТ ТАЗИ ПОМОЩ ЗА ЗАСИЛВАНЕ НА БОРБАТА СРЕЩУ ТРАФИКА НА ЕМИГРАНТИ И ДРУГИ ЛИЦА НА ТЯХНА ТЕРИТОРИЯ И ЗА ЗАСИЛВАНЕ НА КОНТРОЛА ПО ЮЖНАТА ИМ ГРАНИЦА.

\subsubsection{Contraintes du genre discursif}

57 Pour ce dernier cas de figure on doit signaler les emplois spécialisés ${ }^{24}$ dans le corpus MED. Dans ce type d'occurrences, personne est traduit quasi-exclusivement par ПАЦИЕНТ ( patzient «patient »"5, (37) même quand il s'agit d'une locution semi-figée comme dans (38) où personne âgée, qui n'a pas d'équivalent bulgare à proprement parler, est traduit par "patient d'un âge avancé »"

(37)

Effets indésirables très fréquents (peuvent toucher plus d'1 personne sur 10)

МНОГО ЧЕСТИ НЕЖЕЛАНИ РЕАКЦИИ (МОГАТ ДА ЗАСЕГНАТ ПОВЕЧЕ ОТ 1 НА 10 ПАЦИЕНТИ)

(38)

Aucun ajustement posologique n'est nécessaire chez la personne âgée

НЕ Е НЕОБХОДИМО АДАПТИРАНЕ НА ДОЗАТА ПРИ ПАЦИЕНТИТЕ В НАПРЕДНАЛА ВЪЗРАСТ.

Deuxième particularité qui ressort de la comparaison des sous-corpus concerne homme-g et être humain, les deux étant traduits préférentiellement par le pluralia tantum hora. En plus des contraintes de détermination (cf. supra), dans un registre médical, l'emploi des 
deux NHGfr semble réservée aux contextes d'opposition référentielle (notamment avec les classes [-hum], c'est -à-dire des animaux, sujets de tests médicaux) :

(39)

Chez l'animal, il a été démontré que l'époétine alfa, à une dose hebdomadaire d'environ 20 fois supérieure à la dose hebdomadaire recommandée chez l'être humain, diminuait le poids foetal, retardait l'ossification et augmentait la mortalité foetale .

ПРИ ПРОУЧВАНИЯ ВЪРХУ ЖИВОТНИ ЕПОЕТИН АЛФА ПОКАЗВА НАМАЛЯВАНЕ НА ТЕЛЕСНОТО ТЕГЛО НА ФЕТУСА, ЗАБАВЯНЕ НА ПРОЦЕСА НА ОСИФИКАЦИЯ И ПОВИШАВАНЕ НА ФЕТАЛНАТА СМЪРТНОСТ, КОГАТО Е ПРИЛАГАН СЕДМИЧНО В ДОЗИ, ПРИБЛИЗИТЕЛНО 20 ПЪТИ НАД ПРЕПОРЪЧВАНАТА СЕДМИЧНА ДОЗА ПРИ ХОРА.

(40)

La phospholipidose neuronale est survenue chez les chiens traités pour une durée de 3 mois avec des expositions systémiques inférieures à celles obtenues aux doses thérapeutiques chez l'homme.

НЕВРОННА ФОСФОЛИПИДОЗА СЕ НАБЛЮДАВА ПРИ КУЧЕТА, ТРЕТИРАНИ С ДОЗИ 23 МЕСЕЦА, ПРИ ПО-НИСКИ СИСТЕМНИ ЕКСПОЗИЦИИ ОТ ТЕЗИ, НАБЛЮДАВАНИ ПРИ ТЕРАПЕВТИЧНИ ДОЗИ Y XOPA.

59 Enfin, soulignons que si dans le corpus EUR la répartition des NHGfr semble être la plus équilibrée (cf. Figure 1), homme-g et d'être humain apparaissent avant tout dans des structures semi-figées (notamment droits de l'homme, traite des êtres humains). Autrement dit, on peut supposer que dans ce type de discours, la référence à une entité humaine se fait essentiellement avec personne(s) et individu(s), ce qui reste à être vérifié et expliqué le cas échéant.

\section{Conclusion}

Le point de départ de cette étude était un constat plutôt trivial. Compte tenu, d'une part, de la spécificité de la classe référentielle des humains (Schnedecker, 2015) et, d'autre part, de l'existence a priori d'un dispositif lexical semblable de NHG en français et en bulgare, nous nous attendions à un fonctionnement comparable dans les deux langues. Cependant, et sans prétendre avoir épuisé le sujet, au terme de cette étude nous pouvons à la fois dégager quelques spécificités des NHG étudiés respectivement dans les deux langues, et mettre en avant certaines régularités dans les traductions du français vers le bulgare $^{27}$ (cf. Schéma 1 ci-après).

61 En bulgare, les deux NHG qui couvrent le plus large spectre d'emplois sont chovek (subst. « un humain »), pour le singulier, et hora (subst. «gens») pour le pluriel. On peut en déduire qu'il s'agit des deux formes de NHGbg les plus neutres sur le plan sémantique. À cela s'ajoute l'absence d'équivalent lexical du NH français personne, dont la traduction peut se réaliser aussi bien par un NHGbg, que par des $\mathrm{NH}$ spécifiques ou des formes pronominales.

62 La comparaison avec le bulgare s'est également révélée fructueuse en vue d'une meilleure compréhension du français. Aussi, la distinction entre personne et être humain tient à une distinction conceptuelle. Être humain dénote l'individu en tant qu'appartenant à une classe taxinomique (d'où ses emplois dans des contextes sinon en opposition du moins contrastifs avec d'autres classes référentielles) alors que personne opère une « saisie » soit du point de vue « individuel» (ce qui touche au corps et à la personnalité d'un individu) soit du point de vue de son rôle social. Cette piste, cependant demande à être poursuivie 
afin d'expliquer tout le potentiel de la polylexie être humain qui, d'après l'exemple suivant, peut aussi renvoyer à une dimension sensible de l'humain (être humain est traduit par un GV, litt. «Vous n'avez jamais pensé que Carrie a des sentiments ?») :

(41)Vous ne vous êtes donc pas aperçues que Carrie est un être humain?

НЯКОЯ ОТ ВАС ДА СЕ Е ЗАМИСЛЯЛА, НЯКОГА, ЧЕ И КЕРИ УАЙТ ИМА ЧУВСТВА ?

Schéma 1 : Types de contraintes dans la traduction des NHG (FR/BG)
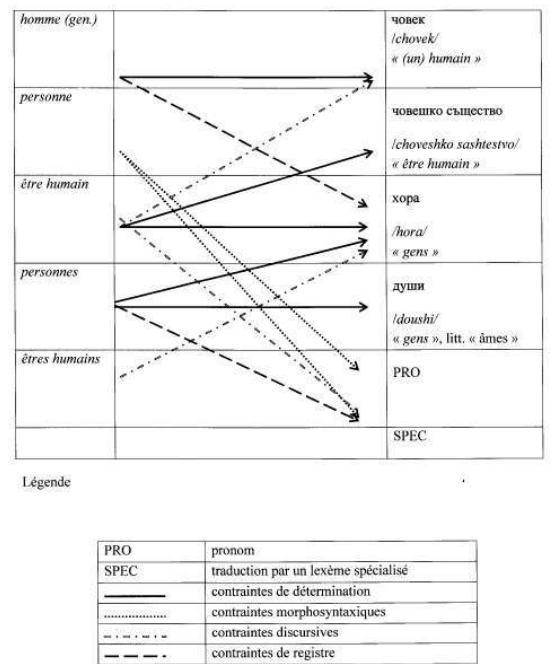

\section{BIBLIOGRAPHIE}

ALEKSANDROVA, A. (2013). Les noms humains de phase : problèmes de classifications ontologiques et linguistiques. Strasbourg, Thèse de Doctorat, Université de Strasbourg.

AleKSANDRova, A., \& MOSTROV, V. (2016). A comparative study of French and Bulgarian human general nouns. Dans SSL-11 (pp. 23-25/sept. 2016). Toronto.

CAPPEAU, P., \& SCHNEDECKER, C. (2014). Gens, personne(s), individu(s). Trois saisies de l'humain. Dans F. Neveu, N. Blumenthal, L. Hriba, A. Gerstenberg, J. Meinschaefer, \& S. Prévost (Éds), CMLF (Congrès Mondial de Linguistique Française) (pp. 3027-3040). Berlin : SHS Web Conférence.

DOBROVIE-SORIN, C. (Éd.). (2005). Noms nus et généricité. Saint-Denis : Presses Universitaires de Vincennes.

GROSS, G. (1996). Les expressions figées en français. Paris : Ophrys. 
KLEIBER, G. (1981). Problèmes de référence: descriptions définies et noms propres. Paris : Klincksieck.

KLEIBER, G. (1989). Généricité et typicalité. Le français moderne, 57, 127-154.

MIHATSCH, W. M (2015). La sémantique des noms génériques « être humain » français et allemands. Dans W. Mihatsch \& C. Schnedecker (Éds), Les noms d'humains : une catégorie à part? (pp. 55-85). Stuttgart : Franz Steiner Verlag.

ROIG, A. (2013). Des N et du N sujets : des généricités toujours réfusées ? Travaux de linguistique, 2

(67), 61-87.

SCHNEDECKER, C. (2015). Un problème à la croisée des disciplines linguistiques : les noms d'humains comme interface entre morphologie, syntaxe et sémantique. Dans A. Rabatel (Éd.), La sémantique et ses interfaces. Actes du colloque bisannuel de l'Association des Sciences du Langage (Vol. octobre, 2013, pp. 111-141). Limoges : Lambert-Lucas.

TIEDERMAnN, J. (2009). News from OPUS - A Collection of Multilingual Parallel Corpora with Tools and Interfaces. Dans N. Nicolov, K. Bontcheva, G. Angelova, \& R. Mitkov (Éds), Recent Advances in Natural Language Processing (Vol. 5, pp. 237-248). Amsterdam/Philadelphia : John Benjamins.

VENKova, V. (1997). The nouns man and people in Portuguese and in Bulgarian. Contrastive Linguistics, 4(22), 5-12.

\section{Dictionnaires}

Le Petit Robert de la langue française (CD-ROM, 2009)

Lexis, Dictionnaire de la langue française, Larousse, Paris, 1998

Le Trésor de la langue française informatisé (http://atilf.atilf.fr/tlf.htm)

Dictionnaire monolingue bulgare (Balgarski talkoven rechnik), Naouka i izkustvo, Sofia 2012

Dictionnaire français-bulgare, Naouka i izkustvo, Sofia 2002

Dictionnaire bulgare-français, Naouka i izkustvo, Sofia 1983

\section{NOTES}

1. Et cela aussi bien dans les dictionnaires français que bulgares.

2. Disponible sur http://opus.lingfil.uu.se/index.php.

3. Corpus EMEA (textes issus de l'Agence européenne des médicaments): http:// opus.lingfil.uu.se/EMEA.php

4. Corpus EUROPARL (textes du Parlement Européen) : http://opus.lingfil.uu.se/Europarl.php

5. Corpus SUBS, textes de sous-titres compilé à partir de http://www.opensubtitles.org/ et disponible sur http://opus.lingfil.uu.se/OpenSubtitles.php

6. Les sous-titrages relèvent plutôt du langage courant, mais il est important de prendre en compte le fait que leur traduction est contrainte par des paramètres spécifiques (entre autres, le temps nécessaire pour la lecture pour une scène donnée).

7. Précisons que les emplois pronominaux de personne, qui feront l'objet d'une étude à part, ne font bien évidemment pas partie de ces chiffres.

8. Le ch se prononce $/ \mathrm{t} \delta /$, le sh se prononce $/ \mathrm{S} /$.

9. Cf. Gross (1996).

10. Comme dans le dictionnaire bilingue consulté les différentes collocations où homme apparaît ne sont pas rangées en fonction de ce qui les unit sémantiquement, nous avons exploité, pour les classer, les subdivisions proposées dans le Petit Robert pour ce NH (d'où les trois grands 
« regroupements »: A, B et C). Notons par ailleurs que (B) et (C) sont regroupés dans le PR, mais nous avons opté pour une distinction entre "qualité » et «fonction » à cause des traductions bulgares qui diffèrent dans chacun de ces deux cas. Nous avons opté pour la même solution concernant la présentation des traductions de personne infra.

11. Pour une étude des conditions de la répartition du sens $+\mathrm{M}$ entre chovek et maj cf. Aleksandrova et Mostrov (2016).

12. Même si les expressions sous (B) et (C) correspondent à homme-m, les qualités ou les fonctions qu'elles dénotent ne sont pas rattachées aux propriétés «saillantes » de l'être humain mâle (sauf peut-être pour homme de guerre), à la différence des contextes qui illustrent HOMME 2.

13. Le PR signale l'expression vieillie une jeune/jolie personne où il s'agit d'une «femme ou jeune fille ».

14. Que l'on peut traduire en français par un être méprisable.

15. Cf. (Cappeau \& Schnedecker, 2012, 2014).

16. D'aucuns pourront nous faire remarquer qu'il existe des ressources lexicographiques numériques qui, dans la mesure où elles sont construites sur des corpus alignés, sont plus proches de l'usage. Cependant, il nous semble que l'utilisation de ce type de ressources est loin d'être optimale, compte tenu du manque justement de caractérisation linguistique. Le lecteur peut se faire son propre avis par exemple à partir de la page web suivante: traduction de personne en bulgare https://bg.glosbe.com/fr/bg/personne.

17. Le singulier dousha (litt. une âme) est perçu comme un terme vieilli ou bien est limité à quelques expressions figées (niama [NEG] jiva [ADJ, " vivante »] dousha [SUBST. «âme »], il n'y a personne).

18. Cf. (Dobrovie-Sorin, 2005 ; Kleiber, 1981, 1989 ; mais voir Roig, 2013 sur la lecture générique via l'indéfini pluriel).

19. Rappelons que les graphiques ne tiennent pas compte de personne-PRO.

20. La traduction, dans ce cas se fera par jena (subst. sg. «femme») et non par chovek (« un humain ») qui, en emploi référentiel, dénote l'être humain mâle.

21. Il semblerait que c'est aussi le cas pour d'autres langues (cf. http://lmsi.net/Droits-de-lhomme-ou-droits).

22. Et non l'interprétation du français en deux types de trafics : le trafic de migrants et le trafic d'êtres humains.

23. Il est bien connu que les termes hypéronymes ne peuvent pas occuper la première place dans une coordination de ce type : *j'ai acheté des fleurs et d'autres roses vs j'ai acheté des roses et d'autres fleurs.

24. Nous donnons l'exemple de patient, mais de toute évidence il ne s'agit pas d'une contrainte du domaine médical puisqu'on note aussi des traductions par potrebitel (« consommateur »), jertva (« victime »), slujitel (« (un) employé »), etc.

25. Notons que l'expression de la fraction dans les deux langues semble ne pas exercer de contrainte au niveau lexical. En bulgare, la traduction de personne en N1 peut toujours se faire par le lexème spécialisé patient : ЕДИН ПАЦИЕНТ НА ДЕСЕТ ХИЛЯДИ.

26. Sur les noms de phase ; cf. (Aleksandrova, 2013). En bulgare, la locution vazrasten chovek (« âgé (un) humain » est sous-déterminée dans la mesure où elle peut désigner aussi bien un individu âgé qu'un individu adulte (pas forcément d'âge avancé).

27. Une étude future appliquera la même méthodologie pour examiner les traductions du bulgare vers le français et approfondir la connaissance sur ce panel de NHG. 


\section{RÉSUMÉS}

Cette étude contrastive français-bulgare des noms d'humains généraux poursuit un double objectif. D'une part, en écho avec le travail de Cappeau \& Schnedecker sur les NHGfr (ici-même), nous caractériserons pour la première fois l'ensemble des NHG existants en bulgare sur le plan morphosyntaxique. D'autre part, en nous appuyant sur l'analyse de corpus parallèles, nous mettrons en évidence les régularités dans la traduction en bulgare de trois NHGfr choisis (homme, personne et être humain) ainsi que les contraintes qui conditionnent le choix lexical/grammatical dans la langue cible (p. ex., personne peut être traduit par « chovek» (humain), « litze » (individu) ou encore par « niakoj » (pr. quelqu'un)).

The aim of this contrastive study that deals with human general nouns (HGN) in French and in Bulgarian is double: first, in connection with Cappeau \& Schnedecker's work on French HGN (in this volume), we characterize for the first time the available Bulgarian HGNs under their morphosyntactic features. Second, using data from parallel corpora, we reveal some clear tendencies in the translation of three French HGNs (homme 'man', personne 'person' and être humain 'human being') that depend on the lexical/grammatical properties of the target language (for example, personne can be translated by "chovek" (man, human), "litze" (person, individual) or by “niakoi” (someone)).

INDEX

Mots-clés : noms généraux d'humains, français, bulgare

\section{AUTEURS}

VASSIL MOSTROV

CALHISTE, Valenciennes

ANGELINA ALEKSANDROVA

4071 EDA, Paris Descartes 